
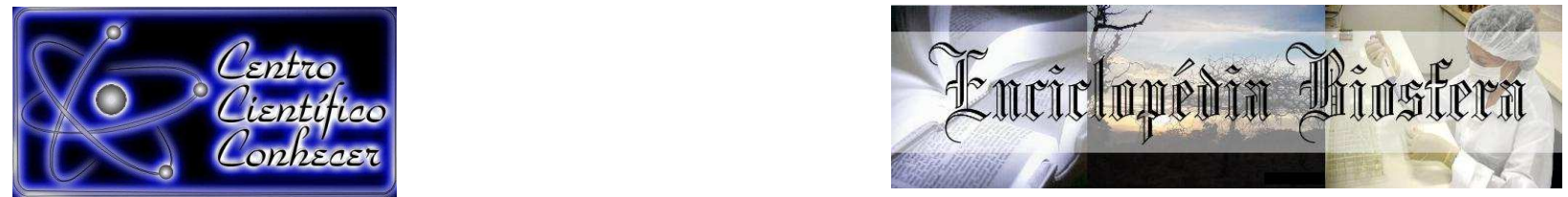

\title{
POTENCIAL DE CRESCIMENTO DE Cordia trichotoma E Grevillea robusta EM PLANTIO PURO E CONSORCIADO
}

Juscelina Arcanjo dos Santos ${ }^{1}$; Rafaella De Angeli Curto²; Diego Fernando Roters; Paulo André Trazzi ${ }^{4}$

${ }^{1}$ Mestre em Ciências Florestais, Universidade Estadual Sudoeste da Bahia (celinarcanjo@hotmail.com), Vitória da Conquista, Bahia

2Professora, Dra., Universidade Federal de Mato Grosso, Campus Universitário de Sinop, Instituto de Ciências Agrárias e Ambientais, Curso de Engenharia Florestal

${ }^{3}$ Mestre em Ciências do Solo, Departamento de Solos e Recursos Naturais, Universidade do Estado de Santa Catarina

${ }^{4}$ Professor, Dr., Instituto Federal de Educação do Norte de Minas Gerais, Departamento de Engenharia Florestal

Recebido em: 02/10/2017 - Aprovado em: 21/11/2017 - Publicado em: 05/12/2017 DOI: 10.18677/EnciBio_2017B50

\begin{abstract}
RESUMO
O estabelecimento de plantios e o acompanhamento do seu potencial de crescimento, por meio de variáveis dendrométricas, torna-se importante ao gerar informações visando avaliar a viabilidade para a implantação e manejo de espécies com características ainda pouco conhecidas. Diante do potencial de uso da madeira das espécies Cordia trichotoma e Grevillea robusta, o objetivo do presente trabalho foi avaliar o potencial de crescimento dessas espécies em plantios puros e consorciados. O experimento foi instalado adotando-se espaçamento entre plantas de $3 \times 3 \mathrm{~m}$, constituído de quatro tratamentos, sendo eles: tratamento 1 = plantio puro de $C$. trichotoma; tratamento 2 = plantio puro de G. robusta; tratamento $3=$ consórcio de $C$. trichotoma e $G$. robusta, com uma linha para cada espécie; e tratamento 4 = consórcio de $C$. trichotoma e $G$. robusta, com as espécies intercaladas nas linhas. Com base na avaliação realizada aos 17 anos em plantio puro e consorciado de $C$. trichotoma e $G$. robusta, foi possível verificar grande variabilidade nas dimensões atingidas. Não houve diferença no crescimento de $C$. trichotoma e G. robusta quando em plantio puro ou consorciado, no entanto a taxa de sobrevivência para $C$. trichotoma tende a diminuir quando em consórcio com a $G$. robusta.
\end{abstract}

PALAVRAS-CHAVE: Dendrometria, Louro-pardo, silvicultura, 


\title{
GROWTH POTENTIAL OF Cordia trichotoma E Grevillea robusta IN PURE AND CONSORTIUM PLANTING
}

\begin{abstract}
The establishment of plantations and the monitoring of their growth potential become important to generat information to evaluate the feasibility for the implantation and management of species with characteristics still little known, mostly through their dendrometric variables. Considering the potential of wood production of the species Cordia trichotoma and Grevillea robusta, the objective of the present work was to evaluate the potential growth of these species in pure and consortium plantations. The experiment was carried out by adopting $3 \times 3 \mathrm{~m}$ plant spacing, consisting of four arrangement: arrangement $1=$ pure planting of $C$. trichotoma; arrangement $2=$ pure planting of $G$. robusta; arrangement $3=$ consortium of $C$. trichotoma and $G$. robusta, with one line for each species; and arrangement $4=$ consortium of $C$. trichotoma and G. robusta, with the species intercalated in the lines. As a basis for the evaluation performed past 17 years of pure planting and intercropping of $C$. trichotoma and $G$. robusta, it was possible to verify a great variability in the dendrometric variables. There was no difference in the growth of $C$. trichotoma and $G$. robusta in pure or consortion plantation, but the survival rate for $C$. trichotoma tends to decrease when in consortium with $G$. robusta.
\end{abstract}

KEYWORDS: Louro-pardo, forestry, dendrometry.

\section{INTRODUÇÃO}

Grande parte das áreas de florestas plantadas para produção de madeira no Brasil utiliza espécies do gênero Eucalyptus e Pinus (IBÁ, 2017), principalmente, em função do mercado já consolidado para os produtos oriundos das diferentes espécies cultivadas destes gêneros, bem como devido a existência de material genético de rápido crescimento e a grande disponibilidade de informações técnicas para o bom desenvolvimento destes plantios. A ausência destes fatores é limitante para que outras espécies, nativas e até mesmo exóticas, sejam pouco usadas em plantios puros e até mesmo consorciados (GONÇALVES et al., 2013).

Assim, o estabelecimento de plantios e o acompanhamento do seu potencial de crescimento, por meio de variáveis dendrométricas, torna-se importante ao gerar informações visando avaliar a viabilidade para a implantação e manejo de espécies com características ainda pouco conhecidas, e que possam ser promissoras em reflorestamentos com interesses econômicos.

Cordia trichotoma (Vell.) Arrab. ex Steud (louro-pardo), espécie arbórea nativa predominantemente da Mata Atlântica brasileira, mesmo apresentando crescimento considerado de lento a moderado (CARVALHO, 1994; SALVADORI et al., 2013), apresenta potencial para plantios com fins econômicos, principalmente devido a excelente qualidade de sua madeira para confecção de móveis de luxo, revestimentos, e lâminas faqueadas para fins nobres, tendo sido muito explorado no passado devido à aceitabilidade de sua madeira no mercado (REITZ et al., 1983; (CARVALHO, 1994; BONNET; CURCIO, 2015).

O louro-pardo apresenta comportamento de espécie secundária inicial a tardia, comportando-se como semi-heliófila tolerante ao sombreamento de média intensidade quando jovem, sendo recomendada para recomposição e recuperação de áreas degradadas (LORENZI, 2002), e podendo ser também combinado com a agricultura durante a primeira fase do crescimento (REITZ et al., 1983). A espécie 
apresenta a habilidade natural de regeneração pelo sistema radicular, constituindo um material com elevado potencial morfogenético (KIELSE et al., 2013).

Grevillea robusta A. Cunn. (grevílea), é uma espécie florestal nativa da Austrália (NATH et al., 2016), que se destaca por apresentar crescimento rápido em condições satisfatórias, plasticidade genética, rusticidade e boa qualidade da madeira, no entanto, apesar de seu potencial, foi introduzida no Brasil com a finalidade de quebra-ventos e sombreamento sobre a cultura do café (FERREIRA; MARTINS, 1998), e mais recentemente sendo indicada para incorporação na produção de painéis aglomerados de Pinus aumentando a estabilidade dimensional dos painéis (TRIANOSKI et al., 2016), caracterizando-se como uma espécie alternativa para reflorestamento, ainda por enriquecer as camadas mais profundas dos solos por meio da ciclagem de nutrientes de suas raízes, contribuindo como fonte de nutrientes para as espécies consorciadas (THAKUR et al., 2015).

Devido à baixa competitividade com as culturas agrícolas (FERREIRA; MARTINS, 1998), G. robusta está entre as mais difundidas em sistemas silvipastoris na região noroeste do Paraná, no entanto, ainda não desempenham todas as suas potencialidades, principalmente porque o potencial madeireiro não é aproveitado economicamente (NEPOMUCENO; SILVA, 2009), fato este relacionado ao desconhecimento de técnicas silviculturais adequadas e do potencial de crescimento.

Diante do potencial de uso da madeira das espécies $C$. trichotoma e $G$. robusta, o objetivo do presente trabalho foi avaliar o potencial de crescimento dessas espécies em plantios puros e consorciados.

\section{MATERIAL E MÉTODOS}

\section{Caracterização da área de estudo}

O presente trabalho foi realizado utilizando dados de um experimento instalado no município de Corupá, Santa Catarina, Fazenda Osvaldo Amaral, localizado nas coordenadas geográficas $26^{\circ} 23^{\prime} 05^{\prime \prime}$ Sul e 49¹6'49" Oeste.

O município de Corupá, que possui uma área de $477 \mathrm{~km}^{2}$, sendo $389,3 \mathrm{~km}^{2}$ da zona rural e $78,70 \mathrm{~km}^{2}$ da zona urbana. O clima, segundo Köppen, é Cfb, subtropical úmido com verão quente, com temperatura média de $18-22^{\circ} \mathrm{C}$, sendo que a máxima atinge os $40^{\circ} \mathrm{C}$ (IBGE, 2017).

\section{Caracterização do experimento}

Para o estabelecimento do experimento com C. trichotoma e G. robusta, foram utilizadas mudas com procedências de Fênix, PR e Cianorte, PR, respectivamente. Foram incorporadas 100 gramas de NPK - 5-20-10 por cova, e para adubação de cobertura realizadas duas aplicações com 40 gramas de ureia no intervalo de 40 dias para $C$. trichotoma, e aplicação com 50 gramas de ureia após 45 dias de plantio para G. robusta.

O experimento foi instalado adotando-se espaçamento entre plantas de $3 \times 3$ $\mathrm{m}$, sendo constituído de quatro tratamentos, sendo eles:

Tratamento 1 = plantio puro de $C$. trichotoma;

Tratamento 2 = plantio puro de G. robusta; 
Tratamento 3 = consórcio de $C$. trichotoma e $G$. robusta, sendo uma linha para cada espécie, e

Tratamento 4 = consórcio de $C$. trichotoma e $G$. robusta, sendo as espécies intercaladas nas linhas.

Cada tratamento foi composto de quatro repetições, com dimensões $24 \times 24$ $\mathrm{m}$, inicialmente constituídas de 64 plantas ocupando $576 \mathrm{~m}^{2}$, totalizando uma área de experimento de aproximadamente $0,92 \mathrm{ha}$.

\section{Análise do crescimento}

Para avaliação do desempenho do crescimento das espécies $C$. trichotoma e G. robusta foram realizadas medições dos diâmetros atingidos pelas plantas e verificadas as taxas de sobrevivência aos 17 anos.

Foi avaliado um delineamento em blocos ao acaso com quatro repetições de quatro tratamentos, sendo os dados submetidos à análise de variância (ANOVA) e a comparação de médias pelo teste de Tukey a 95\% de probabilidade no caso de diferença entre os tratamentos, com auxílio do programa Assistat na versão 7.7 (SILVA; AZEVEDO, 2016).

\section{RESULTADOS E DISCUSSÃO}

\section{Análise dos dados}

Tomando-se como base a avaliação realizada aos 17 anos em plantio puro e consorciado de $C$. trichotoma e $G$. robusta, foi possível verificar grande variabilidade nas dimensões atingidas (Tabela 1).

TABELA 1. Avaliação da variável DAP $(\mathrm{cm})$ e taxa de sobrevivência observada aos 17 anos em plantio puro e consorciado de C. trichotoma e G. robusta

\begin{tabular}{|c|c|c|c|c|c|c|}
\hline Tratamentos com & & & & & & \\
\hline C. trichotoma & Mín. & Máx. & Média & Desvio & IMA & Sobrevivência \% \\
\hline 1 & 4,0 & 25,0 & 12,1 & $\pm 4,6$ & & 66 \\
\hline 3 & 4,0 & 24,0 & 13,0 & $\pm 4,6$ & & 53 \\
\hline 4 & 4,0 & 26,0 & 13,4 & $\pm 5,0$ & & 50 \\
\hline Média & & & 12,8 & & 0,75 & 56,3 \\
\hline $\begin{array}{c}\text { Tratamentos com } \\
\text { G. robusta }\end{array}$ & Mínimo & Máximo & Média & Desvio & IMA & Sobrevivência \% \\
\hline 2 & 3,0 & 27,0 & 10,6 & $\pm 4,6$ & & 75 \\
\hline 3 & 4,0 & 22,0 & 10,8 & $\pm 4,3$ & & 78 \\
\hline 4 & 3,0 & 23,0 & 11,0 & $\pm 4,8$ & & 75 \\
\hline Média & & & 10,8 & & 0,64 & 76,0 \\
\hline
\end{tabular}

A grande amplitude do diâmetro também foi constatada por Radomski et al. (2012) ao avaliarem o crescimento de louro-pardo aos sete anos, em sistemas agroflorestais com espaçamento 2,5 × 2,5 m, em que verificaram altura média de $5,01 \mathrm{~m}( \pm 2,58 \mathrm{~m})$ e diâmetro médio de $8,80 \mathrm{~cm}( \pm 5,02 \mathrm{~cm})$. Os autores atribuem tal 
resultado a não realização de controle genético sobre o material que deu origem às mudas utilizadas para o plantio.

A desuniformidade tanto em altura como em diâmetro, em plantios puros ou não, é fato recorrente em inúmeras espécies florestais nativas (MENDONÇA et al., 2017), no entanto tal desuniformidade também foi verificada em G. robusta. Assim, além da procedência, a falta de manejo adequado, devido ao desconhecimento dos tratamentos adequados para as espécies, levam ao baixo desenvolvimento de suas dimensões na idade avaliada (Tabela 1). Deste modo, verifica-se que a silvicultura dessas espécies ainda é incipiente e necessita de solução para a heterogeneidade de crescimento dos indivíduos plantados.

Em termos de diâmetro médio obtido pelos tratamentos, é possível verificar que independente de ser plantado puro ou consorciado (intercalando linhas ou plantas) não houve diferença estatística no crescimento de $C$. trichotoma $\left(F_{(3,9)}=\right.$ $1,09 ; p=0,35)$ e $G$. robusta $\left(\mathrm{F}_{(3,9)}=1,05 ; p=0,39\right)$, no entanto, é possível observar que a taxa de sobrevivência para $C$. trichotoma tende a diminuir quando em consórcio com a G. robusta.

Em condições de floresta natural, $C$. trichotoma apresentaram DAP médio de apenas $3,6 \mathrm{~cm}$ aos 15 anos e $6,5 \mathrm{~cm}$ aos 20 anos, na depressão central do Estado do Rio Grande do Sul, ao utilizar dados obtidos em análise dendrocronológica (SCHNEIDER et al., 2006), sendo inferior ao incremento observado no presente trabalho. Porém, quando comparado a condições de plantios puros e até mesmo consorciados, tal superioridade deixa de ocorrer.

Em plantios de louro-pardo em arborização de ervais no Rio Grande do Sul foram observados valores médios de diâmetro de $24,41 \mathrm{~cm}$ e $8,39 \mathrm{~m}$ de altura total, aos nove anos de idade, sendo observada a diminuição nos incrementos já entre seis e nove anos de idade (BAGGIO et al., 2011), podendo ser apontado como um provável atingimento da fase de maturidade, tendendo a senescência.

Radomski et al. (2012), em plantios consorciados no Paraná, verificaram IMA de 1,82 cm, aos 10 anos, correspondendo a mais que o dobro observado para 0 presente trabalho. Porém, caso a avaliação fosse realizada sete anos a mais, para se igualar a idade da avaliação do presente estudo, poderia diminuir muito o IMA, principalmente caso venha alcançar a fase de senescência.

Carvalho (1994) relatou que o louro-pardo apresenta crescimento de lento a moderado no Brasil, conforme também pôde ser verificado com o experimento instalado. Porém, G. robusta, considerada espécie de rápido crescimento (FERREIRA; MARTINS, 1998) apresentou diâmetro médio muito baixo. Devido ao rápido crescimento, é possível que o momento de máxima produtividade da $G$. robusta para o local analisado já tenha sido atingido.

Tal fator pode estar associado a sensibilidade da G. robusta ao sombreamento. Radomski et al. (2012), em sistema silvipastoril, observaram que a grevílea foi sensível ao sombreamento, assim como foi observado por Lott et al. (2000) em plantio agroflorestal no Quênia, destacando-se a importância de plantios onde o crescimento das árvores não seja comprometido pela competição. No entanto, mesmo no plantio puro, onde não havia sombreamento ocasionado pela competição com $C$. trichotoma, a $G$. robusta resultou em baixo crescimento. Tal fato pode estar atrelado a mato competição e também a procedência, visto que Fritzsons et al. (2014) verificaram diferença de incremento ao avaliar material de três procedências de $G$. robusta.

Em um ensaio conduzido em Posadas Misiones, Argentina, com 4 densidades de plantio iniciais (162, 375, 750 e 1500 plantas / ha), Barth et al. (2016) verificaram 
que o maior espaçamento resultou em maior diâmetro dos indivíduos, mas a maior acumulação da biomassa do fuste por hectare foi em espaçamentos menores.

Em plantios localizados na Depressão Central do estado do Rio Grande do Sul, aos 17 anos observou-se em média DAP de $25,3 \mathrm{~cm}$ para G. robusta, correspondendo a um IMA de 1,49 cm (CANTO; SCHNEIDER, 2004). As dimensões observadas pelos autores levaram a indicar a espécie para reflorestamentos com 0 objetivo de produção de madeira de grandes dimensões para desdobro ou até mesmo para laminação, devido ao grande potencial de crescimento, além das boas características tecnológicas de sua madeira, porém, utilizaram para a análise apenas árvores dominantes. Nesse sentido, o experimento analisado possui árvores com até $27 \mathrm{~cm}$ de DAP (Tabela 1), demonstrando, neste caso isolado alto potencial de crescimento, o que não pode ser generalizado para indicação da espécie e procedência testada.

\section{CONCLUSÕES}

Não houve diferença no crescimento de $C$. trichotoma e $G$. robusta quando em plantio puro ou consorciado, no entanto, a taxa de sobrevivência para $C$. trichotoma tende a diminuir quando em consórcio com a G. robusta.

O crescimento das espécies avaliadas foi baixo quando confrontado com a literatura, podendo estar associados a seleção de material genético e práticas silviculturais inapropriadas.

\section{REFERÊNCIAS}

BAGGIO, A. J.; FELIZARI, S.R.; RUFFATO, A.; SOARES, A. Produção do componente arbóreo no sistema agroflorestal da erva-mate em Machadinho, RS. Colombo: Embrapa Florestas, 2011. 13 p. Disponível em: https://www.embrapa.br/busca-de-publicacoes/-/publicacao/889661/producao-docomponente-arboreo-no-sistema-agroflorestal-da-erva-mate-ilex-paraguariensis-emmachadinho-rs.

BARTH, S. R.; GIMÉNEZ, A. M.; JOSEAU, M. J.; GAUCHAT, M. E.; FASSOLA, H. E. Compartimentalización de la biomasa aérea de Grevillea robusta A. en función a la densidad inicial de plantación. Scientia Forestalis, v. 44, n. 111, p. 653-664, 2016. Disponível em: DOI: dx.doi.org/10.18671/scifor.v44n111.11.

BONNET, A.; CURCIO, G. R. Uso de espécies arbóreas nativas para a propriedade rural e mercado regional na região de Sooretama, ES. Comunicado Técnico 368, Colombo, PR: Embrapa Florestas, 2015. 16 p. Disponível em: https://www.embrapa.br/busca-de-publicacoes/-/publicacao/1052928/uso-de especies-arboreas-nativas-para-a-propriedade-rural-e-mercado-regional-na-regiaode-sooretama-es

CANTO, J. L.; SCHNEIDER, P. R. Crescimento da Grevillea robusta A. Cunn. na depressão central do Rio Grande do Sul, Brasil. Ciência Florestal, Santa Maria, v. 14, n. 2, p. 29-35, 2004.Disponível em: http://dx.doi.org/10.5902/198050981804.

CARVALHO, P. E. Espécies florestais brasileiras: recomendações silviculturais, potencialidades e usos da madeira. Colombo, Empresa Brasileira de Pesquisa 
Agropecuária, Centro Nacional de Pesquisa de Florestas, 1994. 640p. Disponível em: http://www.cnpf.embrapa.br/pesquisa/efb/temp/index_especies.htm.

FERREIRA, C. A.; MARTINS, E. G. O potencial da grevílea (Grevillea robusta A. Cunn.) para reflorestamento. In: GALVÃO, A. P. M. (Org.). Espécies não tradicionais para plantios com finalidades produtivas e ambientais. Curitiba: Embrapa Florestas, 1998, p. 169-178. Disponível em: http://www.alice.cnptia.embrapa.br/alice/handle/doc/307863 .

FRITZSONS, E.; MATTOS, P. P.; AGUIAR, A. V.; BRAZ, E. M.; GRABIAS, J.; FERRAZ, M. Crescimento da Grevillea robusta em diferentes sítios edafoclimáticos no Estado do Paraná. Scientia Forestalis, Piracicaba, v. 42, n. 103, p. 383-392, 2014. http://www.ipef.br/publicacoes/scientia/leitura.asp?Article=08\&Number=103\&p=n.

GONÇALVES, J. L. M.; ALVARES, C. A.; HIGA, A. R.; SILVA, L. D.; ALFENAS, A. C.; STAHL, J.; FERRAZ, S. F. B.; LIMA, W. P.; BRANCALION, P. H. S.; HUBNER, A.; BOUILLET, J. P. D.; LACLAU, J. P.; NOUVELLON, Y.; EPRO, D. Integrating genetic and silvicultural strategies to minimize abiotic and biotic constraints in Brazilian eucalypt plantations. Forest Ecology and Management, v. 301, p. 6-27, 2013. Disponível em: https://doi.org/10.1016/j.foreco.2012.12.030.

IBÁ - Indústria Brasileira de Árvores: Anuário Estatístico. Brasília, 2017. 80 p.

IBGE - Instituto Brasileiro de Geografia e Estatística. Disponível em https://sidra.ibge.gov.br/bda/pesquisas. Acesso em 09 set. 2017.

KIELSE, P.; BISOGNIN, D.A.; HEBERLE, M.; FLEIG, F. D.; XAVIER, A.; RAUBER, M. A. Propagação vegetativa de Cordia trichotoma (Vell.) Arrab. Exsteudel por estaquia radicular. Revista Árvore, v. 37, p.59-66, 2013. Disponível em: http://dx.doi.org/10.1590/S0100-67622013000100007.

LORENZI, H. Árvores brasileiras: manual de Identificação e cultivos de plantas arbóreas do Brasil. 2. ed. Nova Odessa: Instituto Plantarum; 2002.

LOTT, J. E.; HOWARD, S. B.; ONG, C. K.; BLACK, C. R. Long-term productivity of a Grevillea robusta-based overstorey agroforestry system in semi-arid Kenya: I. Tree growth. Forest Ecology and Management, v. 139, n. 1-3, p. 175-186, 2000. Disponível em: https://doi.org/10.1016/S0378-1127(00)00266-8.

MENDONÇA, G. C.; CHICHORRO, J. F.; MENDONÇA, A. R.; GUIMARÃES, L. A. O. P. Avaliação silvicultural de dez espécies nativas da Mata Atlântica. Ciência Florestal, v. 27, n. 1, p. 277-290, 2017. Disponível em: http://dx.doi.org/10.5902/1980509826466.

NATH, C. D.; SCHROTH, D.; BURSLEM, D. F.R.P. Why do farmers plant more exotic than native trees? A case study from the Western Ghats, India. Agriculture, Ecosystems \& Environment, v. 230, p. 315-328, 2016. Disponível em: https://doi.org/10.1016/j.agee.2016.05.013. 
NEPOMUCENO, A. N.; SILVA, I. C. Caracterização de sistemas silvipastoris da região Noroeste do estado do Paraná. Floresta, v. 39, n. 2, p. 279-287, 2009. Disponível em: http://dx.doi.org/10.5380/rf.v39i2.14556.

RADOMSKI, M. I.; PORFÍRIO-DA-SILVA, V.; CARDOSO, D. C. Louro-pardo (Cordia trichotoma (vell.) Arrab. ex Steud.) em sistemas agroflorestais. Colombo: Embrapa Florestas, 2012. 34 p. (Embrapa Florestas. Documentos, 242). Disponível em: https://www.embrapa.br/busca-de-publicacoes//publicacao/945612/louro-pardo-cordia-trichotoma-vell-arrab-ex-steud-em-sistemasagroflorestais.

REITZ, R.; KLEIN, R. M.; REIS, A. Projeto madeira do Rio Grande do Sul. Porto Alegre: Secretaria de Agricultura e Abastecimento, 1983. 524p.

SALVADORI, S. L.; DUARTE, C. U. N. B. D.; SILVA, A. F. G.; KLEIN, W. L. Análise de sobrevivência e crescimento de Cordia trichotoma, Boraginaceae, Lamiales, no Sul de Mato Grosso do Sul - Brasil. Ciência Florestal, v. 23, p. 735-742, 2013. Disponível em: http://dx.doi.org/10.5902/1980509812357.

SCHNEIDER, P. S. P.; SCHEEREN, L. W.; SCHNEIDER, P. R.; FINGER. C. A. G. Crescimento da Cordia trichotoma (Vell.) Arrab. Ex Steud., na depressão central do Estado do Rio Grande do Sul. Revista Floresta e Ambiente, v.13, n.1, p. 26-33, $2006 . \quad$ Disponível em: http://www.floram.org/articles/view/id/4fedf6291 ef1fa9366000008.

SILVA, F. de A. S.; AZEVEDO, C. A. V. The Assistat Software Version 7.7 and its use in the analysis of experimental data. African Journal of Agricultural Research, v.11, n.39, p.3733-3740, 2016.

THAKUR; S.; KUMAR; B. M.; KUNHAMU, T. K. Coarse root biomass, carbon, and nutrient stock dynamics of different stem and crown classes of silver oak (Grevillea robusta A. Cunn. ex. R. Br.) plantation in Central Kerala, India. Agroforestry Systems, v. 89, p. 869-883, 2015. Disponível em: https://doi.org/10.1007/s10457015-9821-y.

TRIANOSKI, R.; PICCARDI, A. B. R.; IWAKIRI, S.; MATOS, J. L. M.; BONDUELLE, G. M. Incorporação de Grevillea robusta na Produção de Painéis Aglomerados de Pinus. Floresta e Ambiente, v. 23, n. 2, p. 278-285, 2016. Disponível em: http://dx.doi.org/10.1590/2179-8087.141515. 